\title{
Formation of massive gas giants on wide orbits
}

\author{
Eduard I. Vorobyov ${ }^{1,2,3}$ and Shantanu Basu ${ }^{3}$ \\ ${ }^{1}$ Research Institute of Physics, Southern Federal University, Rostov-on-Don, 344090, Russia \\ ${ }^{2}$ The Institute for Computational Astrophysics, Saint Mary's University, Halifax, Canada \\ email: vorobyov@ap.smu.ca \\ ${ }^{3}$ Dept. of Physics and Astronomy, The University of Western Ontario, London, Canada \\ email: basu@uwo.ca
}

\begin{abstract}
We present a mechanism for the formation of massive gas giants on wide orbits via disk fragmentation in the embedded phase of star formation. In this phase, protostellar disks undergo radial pulsations which lead to periodic disk compressions and formation of massive fragments on radial distances of the order of 50-300 AU. The fragments that form during the last episode of disk compression near the end of the embedded phase, when torque from spiral arms become weaker, may survive and mature into massive gas giants. This phenomenon can explain the existence of massive exoplanets on wide orbits is such systems as Fomalhaut and HR 8799.
\end{abstract}

Keywords. instabilities, planetary systems: formation, planetary systems: protoplanetary disks

\section{Introduction}

The recent discoveries of massive gas giants on wide orbits of the order of 50-300 AU around HR 8799 (Marois et al. 2008), Fomalhaut (Kalas et al. 2008), and 1RXS J160929.1210524 (Lafreniére et al. 2010) present a new challenge for the currently most favoured core accretion theory for planet formation, whereby giant planet formation is restricted to the inner 5-10 AU. Planet scattering to larger radii is needed to account for the aforementioned systems but this mechanism does not seem to produce stable and low eccentricity orbits (Dodson-Robinson et al. 2010).

On the other hand, gravitational fragmentation is expected to occur in the outer disk regions at distances of the order of a few tens or hundreds of AU (e.g., Durisen et al. 2007) and thus can naturally explain the in situ formation of wide-orbit extrasolar planets. In this article, we demonstrate that this mechanism is viable and present the results of our numerical hydrodynamics simulations illustrating the formation of a massive gas giant at an orbital distance of 55 AU. We refer the reader to Vorobyov \& Basu (2010) for the in-depth discussion of our results.

\section{Formation of a massive gas giant}

We start our numerical hydrodynamics simulations from a pre-stellar cloud core with mass $M_{\text {core }}=0.9 M_{\odot}$ and ratio of rotational to gravitational energy $\beta=1.3 \times 10^{-2}$. The upper rows of images in Figure 1 present the gas surface density (in $\mathrm{g} \mathrm{cm}^{-2}$ ) in the inner $300 \mathrm{AU}$ at different times $t$ after the formation of a protostellar disk. These images demonstrate that fragmentation occurs early in the disk evolution, already at an age of $0.03 \mathrm{Myr}$. However, at $t=0.14 \mathrm{Myr}$ all fragments are either driven into the star due to exchange of angular momentum with spiral arms or dispersed due to tidal torques. 

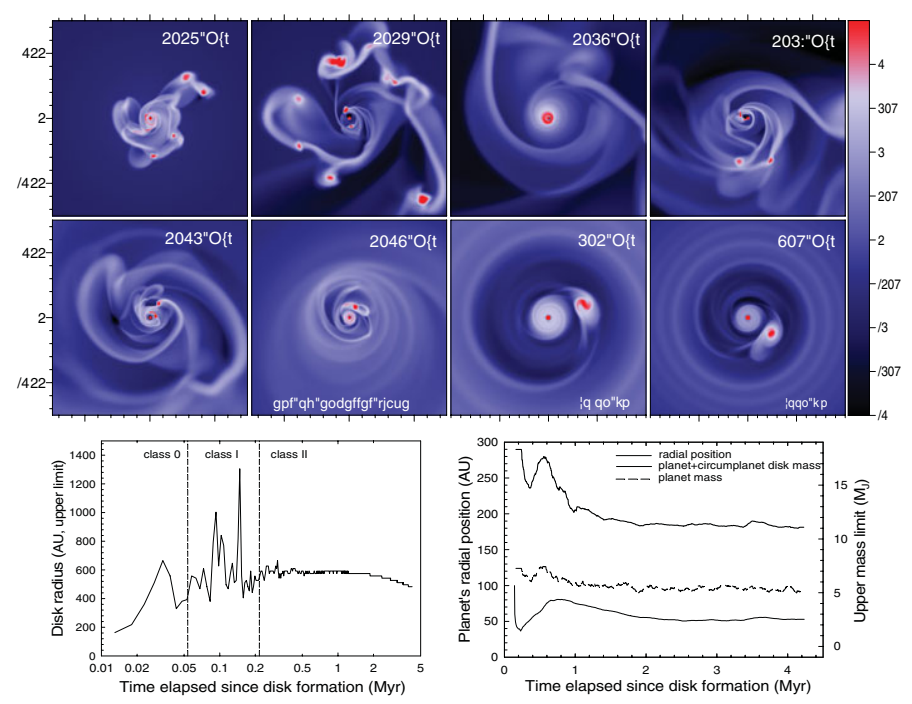

Figure 1. Top two rows. Gas surface density in the inner 300 AU at several distinct times after the disk formation. Bottom-left. Disk radius versus time. Bottom-right. Planet's radial position (thin solid line), net mass (dashed line), and total (planet+mini-disk) mass (thick solid line) versus time.

The inward radial migration of the fragments deposits angular momentum to the outer parts of the disk, which results in periodic disk expansions as shown by the solid line in the bottom-left panel of the Figure. The last episode of expansion, at $t \approx 0.14 \mathrm{Myr}$, is particularly strong and, when followed by disk contraction, it gives birth to a last set of fragments. This happens near the end of the Class I phase (as indicated by the vertical dashed lines) when gravitational and tidal torques from spiral arms are becoming weaker due to diminishing mass infall from the envelope - the major disk destabilizing mechanism. As a result, one of the fragments survives and matures into a massive gas giant at an orbit of $\approx 55 \mathrm{AU}$ (thin solid line in the bottom-right panel). The total (planet plus its mini-disk) mass is about $5 M_{\mathrm{J}}$ (dashed line), while the net planet mass is about $12 M_{\mathrm{J}}$ (thick solid line).

E.I.V. gratefully acknowledges support from an ACEnet Fellowship. Numerical simulations were done on the Atlantic Computational Excellence Network (ACEnet). This project was also supported by RFBR grant 10-02-00278 and by the Ministry of Education grant RNP 2.1.1/1937. S. B. was supported by a Discovery Grant from the Natural Sciences and Engineering Research Council of Canada.

\section{References}

Dodson-Robinson, S. E., Veras, D., Ford, E. B., \& Beichman, C. A. 2010, ApJ, 707, 79

Durisen, R. H., Boss, A. P., Mayer, L., Nelson, A. F., Quinn, T., \& Rice, W. K. M. 2007, in Protostars and Planets V, eds. B. Reipurth, D. Jewitt, \& K. Keil, University of Arizona Press, Tucson, 607

Kalas, P., Graham, J. R., Chiang, E., Fitzgerald, M. P., Clampin, M., Kite, E. S., Stapelfeldt, K., Marois, C., \& Krist, J. 2008, Science, 322, 1345

Lafreniére, D., Jayawardhana, R., \& van Kerkwijk, M. H. 2010, ApJ, 719, 497

Marois, C., Macintosh, B., Barman, T., Zuckerman, B., Song, I., Patience, J., Lafreniére, D., \& Doyon, R. 2008, Science, 322, 1348

Vorobyov, E. I. \& Basu, S. 2006, ApJ, 650, 956

Vorobyov, E. I. \& Basu, S. 2010, ApJL, 714, 133 